\title{
Performance of Fitness Functions Based on Natural Frequencies in Defect Detection Using the Standard PSO-FEM Approach
}

\author{
Xiao-Lin Li, ${ }^{1}$ Roger Serra $\mathbb{D}^{1},{ }^{1}$ and Julien Olivier $\mathbb{D}^{2}$ \\ ${ }^{1}$ INSA Centre Val de Loire, Gabriel Lamé Mechanics Laboratory (LAME) E.A. 7494, 41000 Blois, France \\ ${ }^{2}$ INSA Centre Val de Loire, Laboratoire d'Informatique Fondamentale et Appliquée de Tours (LIFAT-EA6300), \\ 41034 Blois, France \\ Correspondence should be addressed to Roger Serra; roger.serra@insa-cvl.fr
}

Received 26 January 2021; Revised 13 February 2021; Accepted 17 February 2021; Published 3 March 2021

Academic Editor: Chengwei Fei

Copyright (C) 2021 Xiao-Lin Li et al. This is an open access article distributed under the Creative Commons Attribution License, which permits unrestricted use, distribution, and reproduction in any medium, provided the original work is properly cited.

\begin{abstract}
Structural defect detection based on finite element model (FEM) updating is an optimization problem by minimizing the discrepancy of responses between model and measurement. Researchers have introduced many methods to perform the FEM updating for defect detection of the structures. A popular approach is to adopt the particle swarm optimization (PSO) algorithm. In this process, the fitness function is a critical factor in the success of the PSO-FEM approach. Our objective is to compare the performances of four fitness functions based on natural frequencies using the standard PSO-FEM approach for defect detection. In this paper, the definition of the standard PSO algorithm is first presented. After constructing the finite element benchmark model of the beam structure, four commonly used fitness functions based on natural frequencies are outlined. Their performance in defect detection of beam structures will be evaluated using the standard PSO-FEM approach. Finally, in the numerical simulations, the population diversity, success rate, mean iterations, and CPU time of the four fitness functions for the algorithm are calculated. The simulation results comprehensively evaluate their performances for single defect and multidefect scenario, and the effectiveness and superiority of the fitness function $S_{4}$ will be demonstrated.
\end{abstract}

\section{Introduction}

Over the last few years, many methods have been developed in the field of defect detection. There are two existing nondestructive methods for defect detection: the local defect detection method and the global defect detection method [1]. The local defect detection method requires specific prior knowledge of the defect distribution and is based on the assessment of readily accessible areas. However, in the defect detection of engineering structures, the general situation of structural defect is usually unknown before defect detection. Therefore, many global defect detection methods for complex high-dimensional structures are provided using the vibration-based information of the structure. The principal point of defect detection based on dynamic structural changes is that the model parameters of a structure are a function of the physical parameters [2]. Therefore, the presence of defects will cause the model parameters of the structure to change; these changes of undamaged and damaged structures can be utilized to diagnose the location and severity of the defect. Generally, the finite element model (FEM) updating method is employed to address such problems [3-5]. In this process, the fitness function is designed to minimize the discrepancy of the responses between model and measurement, which is a key factor in detecting the location and severity of defects. Natural frequencies, mode shape, and modal curvature structural flexibility [6] of the structure have been used as indicators to form the fitness function. The objective is to search for a specific response parameter for which its predicted dynamic responses are consistent with the measurement ones. Therefore, structural defect detection is an inverse optimization problem.

There are significant limitations when using the traditional optimization methods (such as the Newton method [7] and conjugate gradient method [8]) to solve this 
problem, because their ability to process uncertain information is weak, and these methods usually require the fitness function to be convex and continuously differentiable. In contrast, metaheuristic optimization algorithms do not have these requirements for the fitness function. Moreover, they have strong adaptability to the uncertainty of the data in the calculation. For example, the most used optimization algorithms like the genetic algorithm $[9,10]$, the particle swarm optimization algorithm [11-13], the ant colony algorithm $[14,15]$, and other latest optimization algorithms such as the monarch butterfly optimization [16], earthworm optimization algorithm [17], slime mould algorithm [18], and elephant herding optimization (EHO) [19]. Among them, particle swarm optimization (PSO) is the most popular one for defect identification [20]. This algorithm is getting increasing people's attention because compared with other algorithms, it is simple in concept and easy to implement and requires fewer parameters to adjust and fast convergence speed. When using the PSO algorithm for defect identification, the fitness function is always a critical factor for defect localization and quantification. A wellbehaved fitness function can minimize the discrepancy between the measured and model-predicted data effectively. Although many indicators have been used for defect identification [21, 22], there are many difficulties and limitations in extracting other responses, especially with limited sensors. Thus, many researchers appeal to take the pure natural frequency response as the input indicator to form various fitness functions since it can be measured accurately and inexpensively. However, almost no one is devoted to studying the influences of different fitness functions on the performance of the algorithm, and even there are no uniform evaluation criteria to conduct the performance evaluation of the algorithm for defect detection. Therefore, this paper provides comprehensive evaluation indexes to analyze the performances of various fitness functions composed of natural frequencies using the standard PSO algorithm.

This paper is organized as follows: Section 2 introduces the standard PSO algorithm for defect detection of the beam structures. In Section 3, the finite element benchmark model of the beam structure is constructed, and the element stiffness reduction factor is applied to indicate the location and severity of the defect. Moreover, four commonly used fitness functions based on natural frequencies are outlined. In Section 4, three defect scenarios are simulated by using the standard PSO-FEM approach. The population diversity, success rate, mean iterations, and CPU time are provided to comprehensively evaluate the effectiveness and reliability of each fitness function for single defect and multidefect detection and localization scenarios. Finally, Section 5 concludes this paper and depicts some future works.

\section{Standard Particle Swarm Optimization}

The particle swarm optimization algorithm (PSO) is a population-based optimization algorithm [20]. A fully connected topology is used in the algorithm. In an iterative way, the algorithm tries to improve the initial candidate solution concerning a given measure of quality. A candidate solution equals a so-called particle. In a $D$-dimensional real-valued research space, there is a group of randomly generated particles. The position and velocity of the particles are expressed as $\mathbf{X}_{i}=\left(x_{i 1}\right.$, $\left.x_{i 2}, \ldots, x_{i D}\right)^{T}, \mathbf{V}_{i}=\left(v_{i 1}, v_{i 2}, \ldots, v_{i D}\right)^{T}$, respectively. $i=1,2$, $\ldots, N$ represents the number of particles and $j=1,2, \ldots, D$ represents the dimension. The particle can record the best location that it has experienced, that is, the personal best position $\mathbf{P}_{i}=\left(p_{i 1}, p_{i 2}, \ldots, p_{i D}\right)^{T}$ and the best position that all particles have experienced, namely, the global best position $\mathbf{P}_{g}=\left(p_{g 1}, p_{g 2}, \ldots, p_{g D}\right)^{T}$. In the $t^{\text {th }}$ iteration, the velocity and position of the $j^{\text {th }}$ dimension of the $i^{\text {th }}$ particle are updated as follows:

$$
\begin{aligned}
& v_{i j}^{t+1}=\omega^{t} v_{i j}^{t}+c_{1} r_{1}\left(p_{i j}^{t}-x_{i j}^{t}\right)+c_{2} r_{2}\left(p_{g j}^{t}-x_{i j}^{t}\right), \\
& x_{i j}^{t+1}=x_{i j}^{t}+v_{i j}^{t+1},
\end{aligned}
$$

where $\omega$ is the inertia weight, $c_{1}, c_{2}$ are the acceleration coefficients, and $r_{1}, r_{2}$ are random numbers uniformly distributed between $[0,1]$.

In this paper, the PSO parameters are set as reported in the literature [23]. During the search process, $c_{1}=c_{2}=2$ is fixed. In our case, the position of the particle cannot exceed the limit as $x_{i j} \in[0,1]$. The velocity of each dimension cannot exceed the maximum velocity $v_{\max }$ and set its value equal to the boundary value of the search space $v_{\max }=1$. The inertia weight $\omega$ can be seen in

$$
\omega^{t}=\omega_{\max }-\frac{\omega_{\max }-\omega_{\min }}{t_{\max }} t,
$$

where $t_{\max }$ is the maximum number of iterations, $t$ is the current iteration, $\omega_{\max }=0.9$, and $\omega_{\min }=0.4$. Equations (1) and (2) are called the standard PSO algorithm, and the basic flowchart of the standard PSO algorithm is in Figure 1.

\section{PSO-FEM Formulation and Fitness Function Definition}

A steel cantilever beam with a length of $1 \mathrm{~m}$ is selected for numerical simulations. The material parameters of the $2 \mathrm{D}$ Euler-Bernoulli finite element beam model of the structure are [24] Young modulus $E=2.0 \times 10^{11} \mathrm{~Pa}$ and density $\rho=7850 \mathrm{~kg} \cdot \mathrm{m}^{-3}$. The cross section of the beam is defined by the width $w=2.49 \times 10^{-2} \mathrm{~m}$ and the depth $d p=5.3 \times$ $10^{-3} \mathrm{~m}$. Consider that the computational cost will be seriously expanded with the increase of the number of finite elements and our objective focuses on the performance of different fitness functions. The finite element model selects a fixed thirty equal 2D Euler-Bernoulli cantilever beam elements, as illustrated in Figure 2.

For an undamped structure, assuming that the elastic theory is still satisfied after tiny damage, the structural dynamic eigenvalue equation before and after the defect is given by 


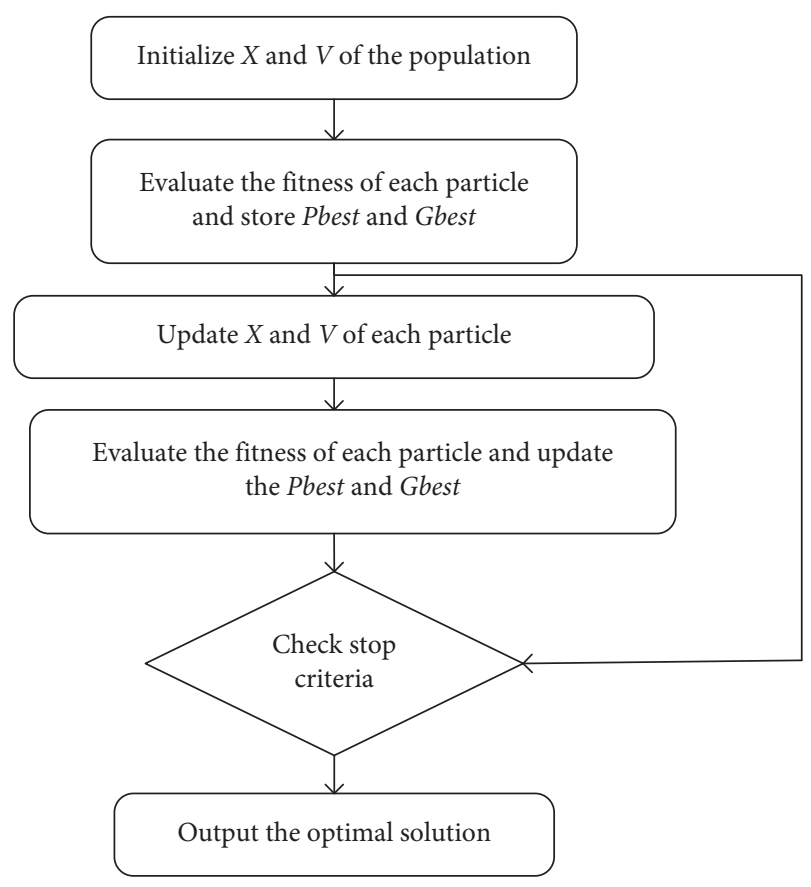

Figure 1: Flowchart of the standard PSO algorithm.

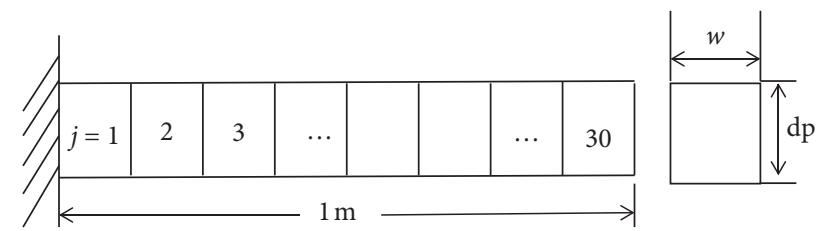

FIgURE 2: The cantilever beam is discretized in 30 Euler-Bernoulli elements.

$$
\begin{gathered}
\left(\mathbf{K}-\omega_{h s}^{2} \mathbf{M}\right) \boldsymbol{\Phi}_{s}=0, \quad(s=1,2, \ldots, m), \\
\left(\mathbf{K}^{\mathrm{d}}-\omega_{d s}^{2} \mathbf{M}\right) \boldsymbol{\Phi}_{s}^{\mathrm{d}}=0, \quad(s=1,2, \ldots, m),
\end{gathered}
$$

where $\mathbf{K}, \mathbf{M}, \omega_{h s}^{2}$, and $\Phi_{s}$ of the intact structure represent the global stiffness matrix, the global mass matrix, the $s^{\text {th }}$ natural frequencies, and the $s^{\text {th }}$ mode shape, respectively. It is assumed that the change in mass before and after the defect is negligible, $\mathbf{K}^{\mathrm{d}}, \omega_{d s}^{2}$, and $\Phi_{s}^{\mathrm{d}}$ still represent the global stiffness matrix, the $s^{\text {th }}$ natural frequencies, and the $s^{\text {th }}$ mode shape, but for the defective structure. In this 2D FEM of the cantilever beam, $\omega_{h s}^{2}$ and $\omega_{d s}^{2}$ can be obtained, respectively, from solving the eigenvalue problems (4) and (5) by using MATLAB eig function. Then, the expression $f_{s}=\left(\omega_{s}\right)$
$2 \pi)(\mathrm{Hz})$ is used to calculate the natural frequencies before and after the defect.

Assuming that the element stiffness of the structure decreases evenly after the defects, the Young modulus reduction factor expressed in the following equation is used to simulate the defect degree of the structure [25]:

$$
\alpha_{j}=\frac{\mathbf{E}-\mathbf{E}_{j}}{\mathbf{E}},
$$

where $E$ and $E_{j}$ are the Young modulus of the $j^{\text {th }}(j=$ $1,2, \ldots, 30)$ element before and after defect. In this condition, the global stiffness matrix $K$ after the defect will be changed as

$$
\mathbf{K}^{d}=\sum_{j=1}^{30}\left(1-\alpha_{j}\right) \mathbf{k}_{j},
$$

where $k_{j}$ denotes the stiffness matrix of the $j^{\text {th }}$ element, $\alpha_{j}$ is the defect severity of the $j^{\text {th }}$ element, and $\alpha=\left(\alpha_{1}, \alpha_{2}\right.$, $\left.\ldots, \alpha_{30}\right)$ represents the defect vector of the structure. The value of $\alpha_{j}$ is between 0 and 1 , when $\alpha_{j}=0$ means the $j^{\text {th }}$ element of the structure is intact, and $\alpha_{j}=1$ means the $j^{\text {th }}$ element of the structure has been completely damaged. Then, after choosing the suitable dynamic responses (like natural frequencies, modal shape, etc.) as indicators, the fitness function can be constructed exactly.

The numerical equation (6) forms the basis of defect simulation through an inverse procedure. In this study, the numerical responses of the damage vector $\alpha$ calculated by the FEM are used as the measurement responses. Our objective is to search for a specific damage vector $\mathbf{X}_{i}$ for which its predicted dynamic responses are consistent with the measurement ones. Therefore, the FEM-based defect detection is an inverse optimization problem, which can be solved by minimizing a fitness function $S\left(\mathbf{X}_{i}\right)$, expressed as follows:

$$
\begin{aligned}
& \arg \min S\left(\mathbf{X}_{i}\right), \quad \text { where } \mathbf{X}_{i}=\left(x_{i 1}, x_{i 2}, \ldots, x_{i 30}\right), \\
& \text { subject to } x_{i j} \in[0,1], \quad(j=1,2, \ldots, 30) .
\end{aligned}
$$

Consider that the natural frequencies are relatively easy to obtain and the test accuracy is high, many researchers use it as an input indicator of structural defect detection, and various algebraic expressions related to it are applied to form the fitness function. However, a few researchers are devoted to studying the effect of different forms of the fitness function composed of natural frequencies on the algorithm performance.

In this paper, four commonly used fitness functions based on natural frequencies will be evaluated and they are introduced as follows, respectively $[11,26,27]$ and [28]: 


$$
\begin{aligned}
S_{1}\left(\mathbf{X}_{i}\right)= & \sum_{s=1}^{m}\left(\frac{\left(f_{s}^{d}\right)^{2}-\left(f_{s i}^{p}\right)^{2}}{\left(f_{s}^{d}\right)^{2}}\right)^{2}, \\
S_{2}\left(\mathbf{X}_{i}\right)= & \sum_{s=1}^{m} \frac{\left(f_{s}^{d}-f_{s i}^{p}\right)^{2}}{\left(f_{s}^{d}\right)^{2}}, \\
S_{3}\left(\mathbf{X}_{i}\right)= & \sqrt{\frac{1}{m} \sum_{s=1}^{m}\left(\frac{f_{s}^{d}}{f_{s i}^{p}}-1\right)^{2},} \\
S_{4}\left(\mathbf{X}_{i}\right)= & -\frac{1}{2}\left[\frac{\left|\Delta \mathbf{F}^{T} \cdot \boldsymbol{\delta} \mathbf{F}\left(\mathbf{X}_{i}\right)\right|^{2}}{\left(\Delta \mathbf{F}^{T} \cdot \Delta \mathbf{F}\right)\left(\boldsymbol{\delta} \mathbf{F}^{T}\left(\mathbf{X}_{i}\right) \cdot \boldsymbol{\delta} \mathbf{F}\left(\mathbf{X}_{i}\right)\right)}\right. \\
& \left.+\frac{1}{m} \sum_{s=1}^{m} \frac{\min \left(f_{s}^{d}, f_{s i}^{p}\right)}{\max \left(f_{s}^{d}, f_{s i}^{p}\right)}\right],
\end{aligned}
$$

where $m$ is the order of the natural frequencies used. There are many options for its value in the relevant literature since the natural frequencies of the structure are easy to measure with high accuracy. However, to avoid different $m$ values from interfering with the performance evaluation of the four fitness functions, it is uniformly set to 9 here. The intact $\mathbf{F}_{h}=$ $\left(f_{1}, f_{2}, \ldots, f_{9}\right)^{T}$ frequency vector and the defective $\mathbf{F}_{d}=$ $\left(f_{1}^{d}, f_{2}^{d}, \ldots, f_{9}^{d}\right)^{T}$ frequency vector of the structure are calculated by the finite element method (FEM) as the measured natural frequencies, and $f_{s}^{d}$ is the $s^{\text {th }}(s=1,2$, $\ldots, 9)$ component of the defective frequency vector $\mathbf{F}_{d}$. $\mathbf{F}\left(\mathbf{X}_{\mathbf{i}}\right)=\left(f_{1 i}^{p}, f_{2 i}^{p}, \ldots, f_{9 i}^{p}\right)^{T}$ is the frequency vector predicted by the standard PSO-FEM approach using the location vector of the particle $\mathbf{X}_{i}(i=1,2, \ldots, 30)$ in the standard PSO algorithm (see in Figure 3), and $f_{s i}^{p}$ is the $s^{\text {th }}$ component of the predicted frequency vector $\mathbf{F}\left(\mathbf{X}_{i}\right)$. In addition, for the fourth fitness function $S_{4}, \Delta \mathbf{F}=\left(\mathbf{F}_{h}-\right.$ $\left.\mathbf{F}_{d} / \mathbf{F}_{h}\right)$ is the change of the frequency vector of the defective structure relative to the frequency vector of healthy structure. $\Delta \mathbf{F}\left(\mathbf{X}_{i}\right)$ is defined as $\Delta \mathbf{F}\left(\mathbf{X}_{i}\right)=\left(\mathbf{F}_{h}-\mathbf{F}\left(\mathbf{X}_{i}\right) / \mathbf{F}_{h}\right)$ is the change of the frequency vector of the standard PSO-FEM model with regard to the frequency vector of healthy structure.

The fitness functions $S_{1}, S_{2}, S_{3}$, and $S_{4}$ are constructed based on the rate of change of frequency. The minimum value for $S_{1}, S_{2}$, and $S_{3}$ is then 0 when the solution is found. However, for the fitness function $S_{4}$, when its value equals -1 , the optimal solution is found, which is distinct from the fitness functions $S_{1}, S_{2}$, and $S_{3}$. When the frequency vector $\mathbf{F}\left(\mathbf{X}_{i}\right)=\mathbf{F}_{d}$, it means the frequency vector predicted by the standard PSO-FEM approach becomes the same as the defective frequency vector $\mathbf{F}_{d}$; at this time, the fitness functions $S_{1}, S_{2}, S_{3}$, and $S_{4}$ are all being minimized, and the optimal solution is obtained.

\section{Numerical Simulations and Discussion}

The numerical simulations for defect detection in a cantilever beam have been performed with four different fitness functions based on natural frequencies, as described in the previous section. Three small scenarios are set as defect cases with different defect locations and defect elements severity, as shown in Table 1: single defect, neighbor defects, and multiple defects.

Moreover, the first nine natural frequencies for the healthy beam and three defect cases in Table 2 are adopted to calculate the fitness function.

The maximum number of iterations is set to 200 since this is enough to ensure the convergence of the standard PSO algorithm for the defect cases in Table 1. Considering that the algorithm may fall into the local optimum, we conduct 100 trials for each defect case so that a relatively stable performance of the algorithm can be obtained. When the optimal solution is obtained for one fitness function from one trial (i.e., $\exists \mathbf{X}_{i}$, s.t. $S_{1}=0, S_{2}=0, S_{3}=0$, or $\left.S_{4}=-1\right)$, the calculation is terminated to save computational time. For cases A, B, and C, the population size is set to 50, 75 , and 100 , respectively [11].

When solving the optimization problem, it is necessary to control the population diversity of the algorithm so that it can search more widely in the solution space. The population standard deviation (psd) used in [29] can be adopted to measure the population diversity of the algorithm affected by the fitness function:

$$
\operatorname{psd}=\sqrt{\frac{\left[\sum_{i=1}^{N} \sum_{j=1}^{D}\left(x_{i j}-\bar{x}_{j}\right)^{2}\right]}{(N-1)}},
$$

where $N, D$, and $\bar{x}$ are the population size, the number of dimensions, and the mean position of the particles, respectively. The change of the psd value can represent different levels of population diversity. When the psd value is small, the swarm has been concentrated in a particular area with low diversity. A larger psdvalue represents higher population diversity. However, the performance of the algorithm cannot be determined solely by the psd value but must be combined with the specific state of the particle.

The results of the psdcomparisons of four fitness functions for the three defect cases are plotted in Figure 4. It can be seen that, as the number of iterations increases, the population diversity of the four fitness functions decreases. In the early stage, the fitness functions $S_{1}, S_{2}$, and $S_{3}$ perform the same level of population diversity, while the fitness function $S_{4}$ has the highest population diversity. However, in the latter stage, the population of the fitness function $S_{3}$ always remains the highest diversity, and the population diversities of the fitness functions $S_{1}$ and $S_{2}$ show competitiveness. In contrast, the fitness function $S_{4}$ significantly 


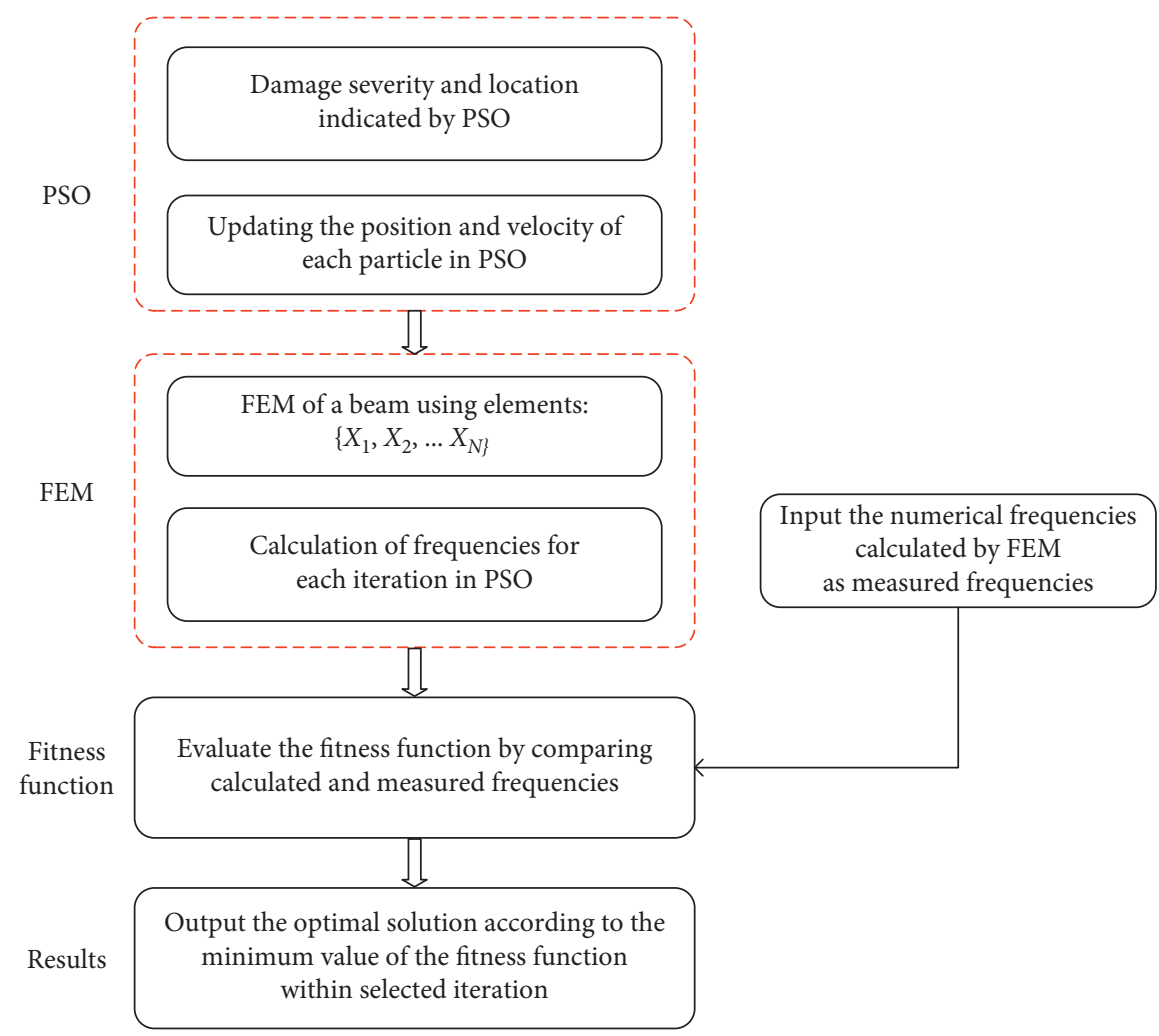

Figure 3: The methodology for defect detection using the standard PSO-FEM approach.

TABle 1: Defect cases of the cantilever beam.

\begin{tabular}{lccc}
\hline Cases & Types & Elements & Severity \\
\hline A & Single defect & 5 & $10 \%$ \\
B & Neighbor defects & 5,6 & $10 \%, 10 \%$ \\
C & Multiple defects & $5,6,25$ & $10 \%, 20 \%, 10 \%$ \\
\hline
\end{tabular}

TAвLE 2: First nine natural frequencies of the cantilever beam $(\mathrm{Hz})$.

\begin{tabular}{|c|c|c|c|c|c|c|c|c|c|c|}
\hline Mode & & $1^{\text {st }}$ & $2^{\text {nd }}$ & $3^{\text {rd }}$ & $4^{\text {th }}$ & $5^{\text {th }}$ & $6^{\text {th }}$ & $7^{\text {th }}$ & $8^{\text {th }}$ & $9^{\text {th }}$ \\
\hline$F_{h}$ & & 4.3215 & 27.0825 & 75.8320 & 148.6018 & 245.6547 & 366.9808 & 512.5964 & 682.5270 & 876.8127 \\
\hline \multirow{3}{*}{$F_{d}$} & Case A & 4.3015 & 27.0652 & 75.8226 & 148.3884 & 244.9609 & 365.8909 & 511.6364 & 682.1218 & 876.5873 \\
\hline & Case B & 4.2839 & 27.0609 & 75.7680 & 148.0051 & 244.2443 & 365.3559 & 511.5336 & 681.6345 & 874.4623 \\
\hline & Case C & 4.2622 & 27.0418 & 75.5330 & 146.9672 & 242.4664 & 364.0223 & 511.2618 & 680.6097 & 869.8996 \\
\hline
\end{tabular}

has the lowest diversity. The high diversity in the early stage means that the population can explore a broader region to find a better potential optimal area, while a rapidly decreasing diversity in the latter stage means that the population can converge fast to the optimal solution. Therefore, we can conclude that the fitness functions $S_{1}$ and $S_{2}$ are competitive in population diversity performance, and the population due to the fitness function $S_{4}$ shows the best diversity performance. Conversely, the population due to the fitness function $S_{3}$ displays the worst diversity performance.

Other performances of the algorithm due to the different fitness functions were also considered, and the relevant results are recorded in Table 3. Here, the success rate is the most important one as it indicates the ability of the algorithm to find the optimal solution. Its value equals the ratio of the number of successful trials among 100 total ones. When $S_{1}=0, S_{2}=0, S_{3}=0$, and $S_{4}=-1$, the optimal solution of the fitness function is obtained, and the defect of the cantilever beam is successfully detected. The variations of the four fitness functions of the three defect cases for 100 trials can be seen clearly in Figure 5. The measure of mean iterations is the average number of iterations needed to reach the optimal solution. For the four fitness functions, 100 trials are launched sequentially, and the CPU time is the time needed to complete 100 trials for each fitness function, which will be recorded in seconds directly during the experiment. The simulation environment is CPU: Inter(R) Core(TM)i5-7600 CPU @ 3.50 GHz. RAM: 16.0 GB. 


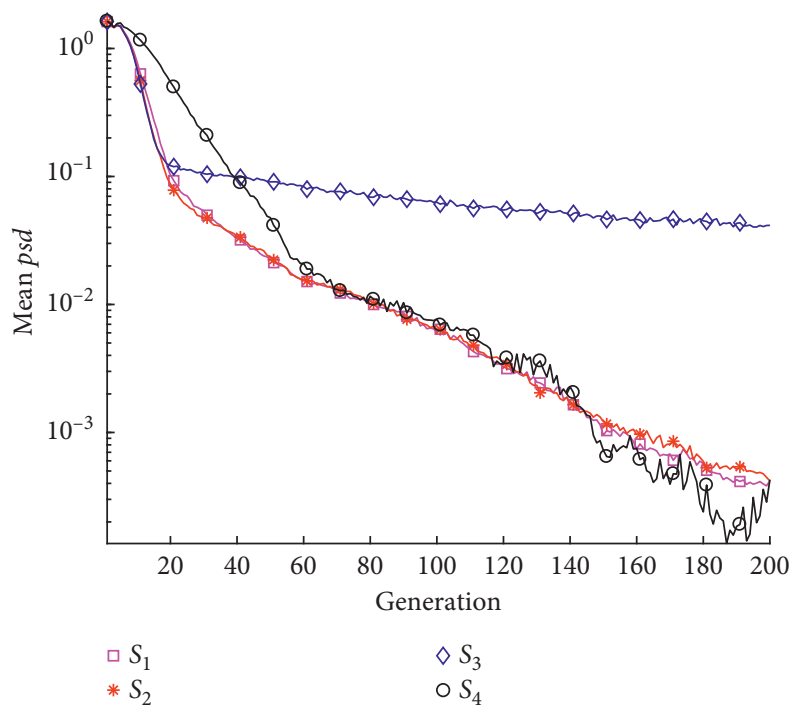

(a)

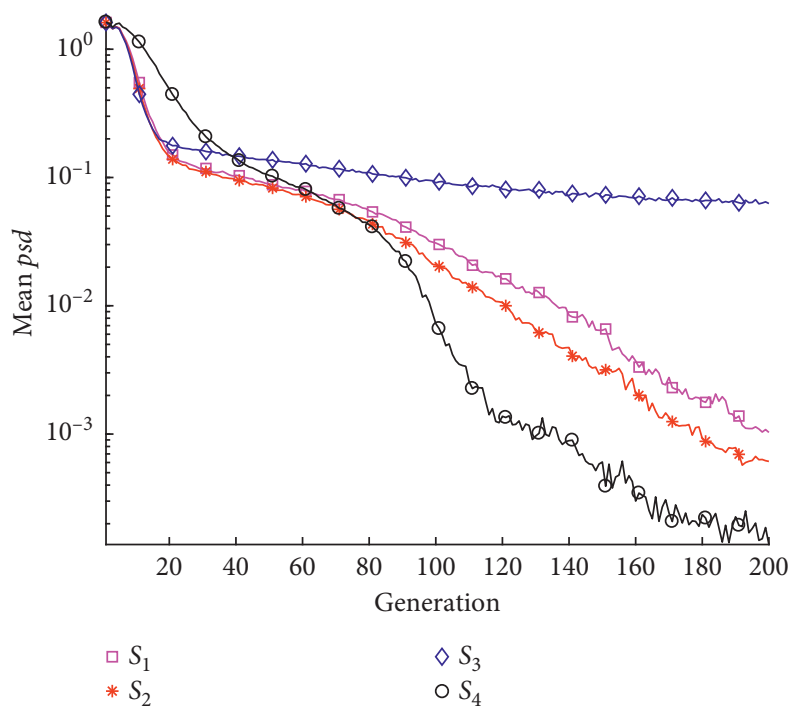

(b)

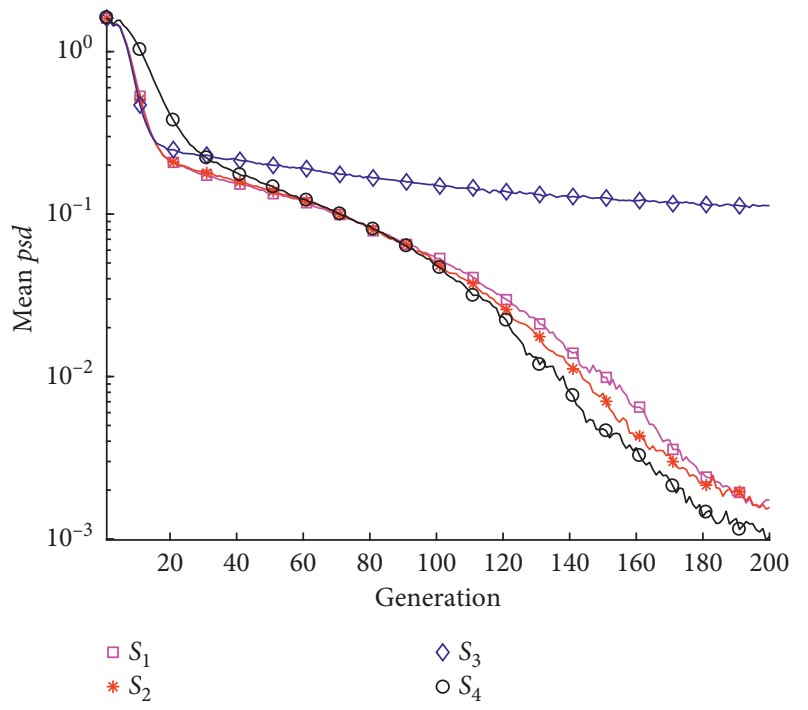

(c)

Figure 4: Mean psd during the run time of four fitness functions for three defective cases. (a) Case (A). (b) Case (B). (c) Case C.

TABLE 3: Evaluation of the performances of four fitness functions for three defect cases.

\begin{tabular}{|c|c|c|c|c|c|}
\hline \multirow{2}{*}{\multicolumn{2}{|c|}{ Damage case }} & \multicolumn{4}{|c|}{ Fitness function } \\
\hline & & $S_{1}$ & $S_{2}$ & $S_{3}$ & $S_{4}$ \\
\hline \multirow{3}{*}{ Case A } & Success rate & 0.5 & 0.49 & 0.03 & 0.97 \\
\hline & Mean iterations & 37.4800 & 37.1224 & 39 & 46.1443 \\
\hline & CPU time & 234.5140 & 232.2411 & 366.1968 & 101.1231 \\
\hline \multirow{3}{*}{ Case B } & Success rate & 0.23 & 0.4 & 0 & 0.96 \\
\hline & Mean iterations & 92.6522 & 90.5250 & $\mathrm{NaN}$ & 89.8125 \\
\hline & CPU time & 494.0094 & 429.0095 & 548.8929 & 270.3995 \\
\hline \multirow{3}{*}{ Case C } & Success rate & 0.09 & 0.08 & 0 & 0.28 \\
\hline & Mean iterations & 139.7778 & 126.1250 & $\mathrm{NaN}$ & 120.0000 \\
\hline & CPU time & 730.5601 & 743.1865 & 766.6580 & 718.9975 \\
\hline
\end{tabular}



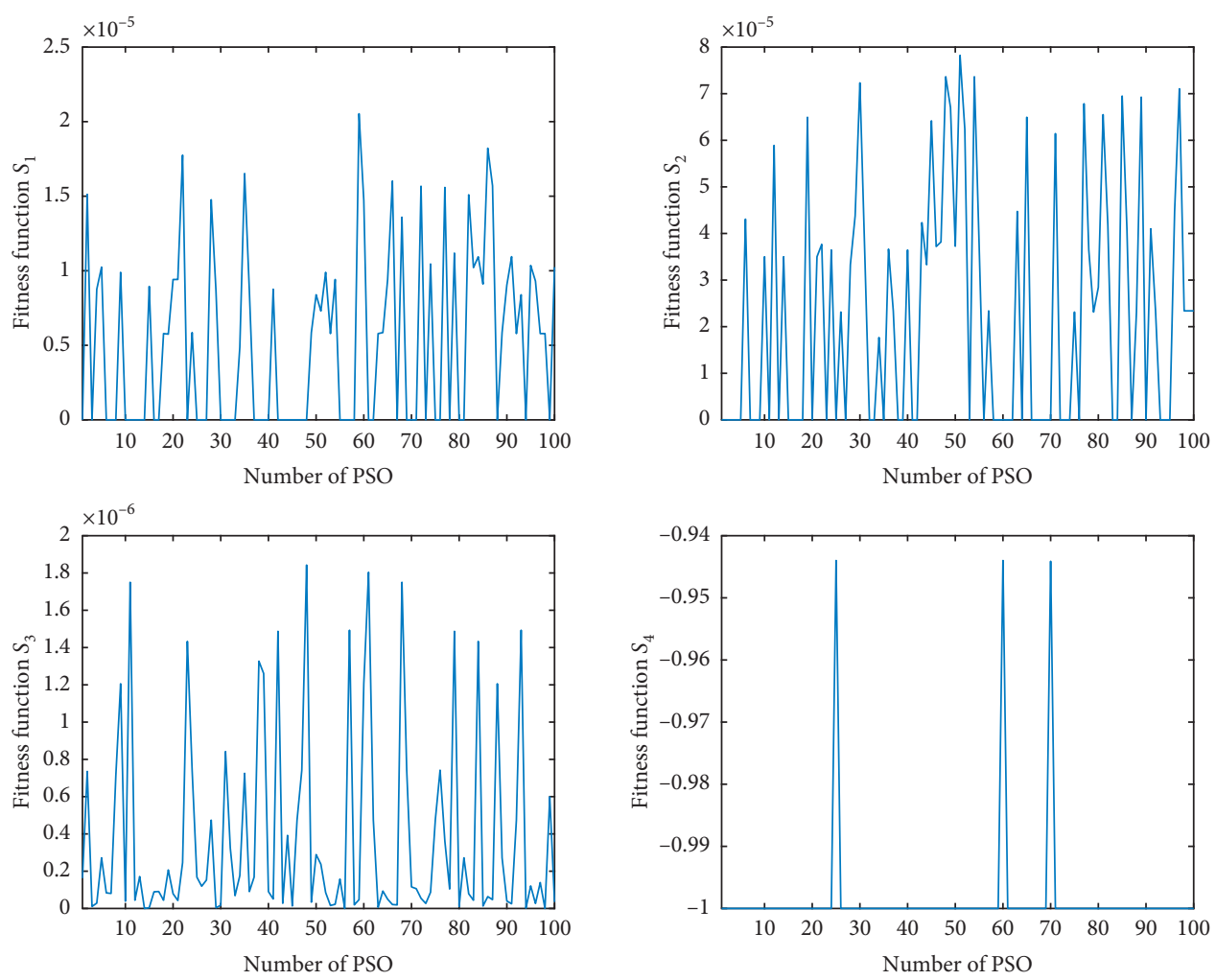

(a)
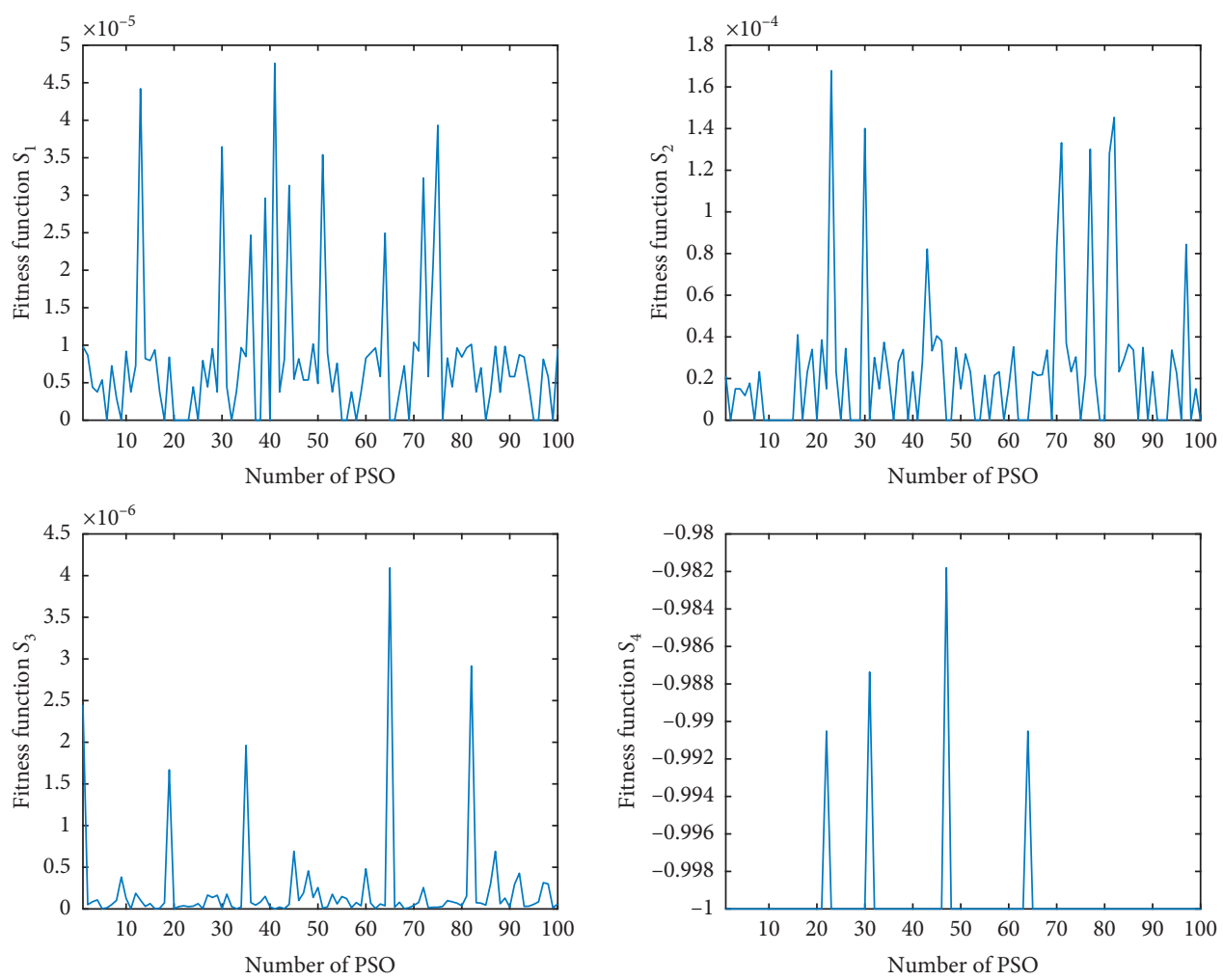

(b)

FIgUre 5: Continued. 

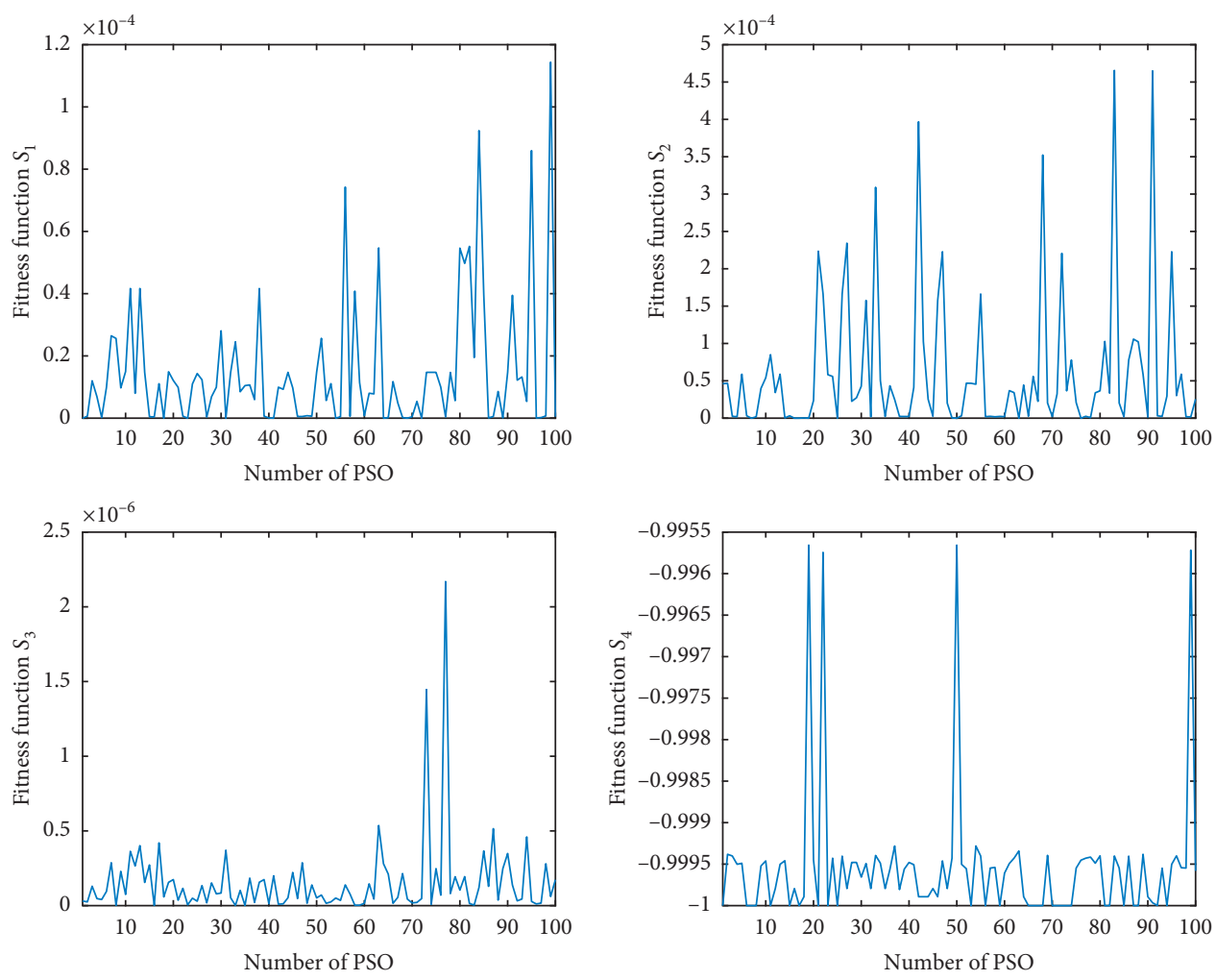

(c)

Figure 5: Variation of the fitness functions $S_{1}, S_{2}, S_{3}$, and $S_{4}$. (a) Case (A). (b) Case (B). (c) Case C.

Combining the results for the three defect cases in Figure 5 and Table 3 , it is visible that the fitness function $S_{4}$ outperforms the fitness functions $S_{1}, S_{2}$, and $S_{3}$ on the success rate, which is the most important indicator for performance evaluation. Correspondingly, it also takes the shortest CPU time due to the highest success rate. The optimal values of the fitness functions $S_{1}, S_{2}$, and $S_{3}$ are all 0 ; comparing the fitness values in Figure 5, it can be obtained that the magnitude of the fitness function $S_{3}$ is lower than $S_{1}$ and $S_{2}$. However, the success rate of $S_{3}$ in Table 3 for defect case B and case C is 0 , which means the algorithm with $S_{3}$ can easily fall into the local optimum and lose its ability to reach the optimal solution. Even for the single defect case A, it also shows the lowest success rate. From the results in Table 3, we can also get that the mean iterations of the fitness function $S_{4}$ are less than the fitness functions $S_{1}, S_{2}$, and $S_{3}$, except for the single defect case. The fitness functions $S_{1}$ and $S_{2}$ are competitive regarding the success rate and the mean iterations. The CPU time for the fitness function $S_{1}$ is slightly better than for the fitness function $S_{2}$. In summary, we can conclude that the algorithm using the fitness function $S_{4}$ performs the best performance. The algorithm shows the worst performance with the fitness function $S_{3}$. The algorithm performances of the fitness functions $S_{1}$ and $S_{2}$ are better than $S_{3}$ but worse than $S_{4}$.

\section{Conclusions}

In this work, the performances of four commonly used fitness functions based on natural frequencies in defect detection are evaluated using the standard PSO-FEM approach. Three damage scenarios with different defect locations and defect element severity are simulated. Four evaluation indexes (population diversity, success rate, mean iterations, and CPU time) are provided for each defect scenario to comprehensively compare the performances of four fitness functions in defect identification. From the simulation results, it can be concluded that the fitness function $S_{3}$ performs the worst performance. The fitness functions $S_{1}$ and $S_{2}$ generally show the same level of performance. The effectiveness and superiority of the best fitness function $S_{4}$ have been significantly illustrated.

In future work, the fitness function $S_{4}$ can be preferentially selected to solve the defect detection of the structures. Besides, the evaluation indexes provided in the paper can be applied to various research fields to analyze the performance of the algorithm comprehensively.

\section{Data Availability}

Data will be available upon request to the authors. 


\section{Conflicts of Interest}

The authors declare that they have no conflicts of interest.

\section{Acknowledgments}

This project was financially supported by the program of the China Scholarship Council (no. 201801810100).

\section{References}

[1] G. W. Housner, L. A. Bergman, T. K. Caughey et al., "Structural control: past, present, and future," Journal of Engineering Mechanics, vol. 123, no. 9, pp. 897-971, 1997.

[2] C. Lu, C.-W. Fei, H.-T. Liu, H. Li, and L.-Q. An, "Moving extremum surrogate modeling strategy for dynamic reliability estimation of turbine blisk with multi-physics fields," Aerospace Science and Technology, vol. 106, pp. 106-112, 2020.

[3] J. M. W. Brownjohn, P.-Q. Xia, H. Hao, and Y. Xia, "Civil structure condition assessment by FE model updating:," Finite Elements in Analysis and Design, vol. 37, no. 10, pp. 761-775, 2001.

[4] R. Serra and L. Lopez, "Damage detection methodology on beam-like structures based on combined modal wavelet transform strategy," Mechanics \& Industry, vol. 18, no. 8, p. 807, 2017.

[5] C.-W. Fei, H.-T. Liu, S. Li, H. Li, L.-Q. An, and C. Lu, "Dynamic parametric modeling-based model updating strategy of aeroengine casings," Chinese Journal of Aeronautics, vol. 101, 2021.

[6] F. Wei and P. Z. Qiao, "Vibration-based damage identification methods: a review and comparative study," Structural Health Monitoring, vol. 10, no. 1, pp. 83-111, 2010.

[7] E. Sachs, "Newton's method for singular constrained optimization problems," Applied Mathematics \& Optimization, vol. 11, no. 1, pp. 247-276, 1984.

[8] S. Djordjevic, "New hybrid conjugate gradient method as a convex combination of LS and CD methods," Filomat, vol. 31, no. 6, pp. 1813-1825, 2017.

[9] J. H. Xie, "Structural damage detection based on fuzzy LSSVM integrated quantum genetic algorithm," Applied Mechanics and Materials, vol. 20-23, pp. 1365-1371, 2010.

[10] J.-H. Chou and J. Ghaboussi, "Genetic algorithm in structural damage detection," Computers \& Structures, vol. 79, no. 14, pp. 1335-1353, 2001.

[11] B. Nanda, D. Maity, and D. K. Maiti, "Vibration based structural damage detection technique using particle swarm optimization with incremental swarm size," International Journal of Aeronautical and Space Sciences, vol. 13, no. 3, pp. 323-331, 2012.

[12] J. Zhao, X. Wu, Q. Sun, and L. Zhang, "Optimal sensor placement for A truss structure using particle swarm optimisation algorithm," The International Journal of Acoustics and Vibration, vol. 22, no. 4, 2017.

[13] G. Ghodrati Amiri, A. Zare Hosseinzadeh, and S. A. Seyed Razzaghi, "Generalized flexibility-based model updating approach via democratic particle swarm optimization algorithm for structural damage prognosis," International Journal of Optimization in Civil Engineering, vol. 55, no. 4, pp. 445-464, 2015.

[14] A. Majumdar, B. Nanda, D. K. Maiti, and D. Maity, "Structural damage detection based on modal parameters using continuous ant colony optimization," Advances in Civil Engineering, vol. 2014, Article ID 174185, 14 pages, 2014.
[15] A. Majumdar, D. K. Maiti, and D. Maity, "Damage assessment of truss structures from changes in natural frequencies using ant colony optimization," Applied Mathematics and Computation, vol. 218, no. 19, pp. 9759-9772, 2012.

[16] G.-G. Wang, S. Deb, and Z. Cui, "Monarch butterfly optimization," Neural Computing and Applications, vol. 31, pp. 1995-2014, 2015.

[17] G.-G. Wang, S. Deb, and L. D. S. Coelho, "Earthworm optimisation algorithm: a bio-inspired metaheuristic algorithm for global optimisation problems," International Journal of Bio-Inspired Computation, vol. 1, no. 1, 2015.

[18] S. Li, H. Chen, M. Wang, A. A. Heidari, and S. Mirjalili, "Slime mould algorithm: a new method for stochastic optimization," Future Generation Computer Systems, vol. 111, pp. 300-323, 2020.

[19] G.-G. Wang, S. Deb, and L. D. S. Coelho, "Elephant herding optimization," in Proceedings of the 2015 3rd International Symposium on Computational and Business Intelligence (ISCBI 2015), Bali, Indonesia, December 2016.

[20] J. Kennedy and R. Eberhart, "Particle swarm optimization," in Proceedings of the ICNN95 - International Conference on Neural Networks, Perth, Australia, December 1995.

[21] F. Shabbir, M. Khan, N. Ahmad, M. Tahir, N. Ejaz, and J. Hussain, "Structural damage detection with different objective functions in noisy conditions using an evolutionary algorithm," Applied Sciences, vol. 7, no. 12, p. 1245, 2017.

[22] X. Chen and L. Yu, "Flexibility-based objective functions for constrained optimization problems on structural damage detection," Advanced Materials Research, vol. 186, pp. 383387, 2011.

[23] Y. Shi and R. Eberhart, "Empirical study of particle swarm optimization," in Proceedings of the 1999 Congress on Evolutionary Computation-CEC99 (Cat. No. 99TH8406), Washington, DC, USA, July 1999.

[24] G. Gautier, J.-M. Mencik, and R. Serra, "A finite elementbased subspace fitting approach for structure identification and damage localization," Mechanical Systems and Signal Processing, vol. 58-59, pp. 143-159, 2015.

[25] S. Khatir, I. Belaidi, R. Serra, M. A. Wahab, and T. Khatir, "Republished Paper. Numerical study for single and multiple damage detection and localization in beam-like structures using BAT algorithm," Journal of Vibroengineering, vol. 20, no. 1, pp. 811-822, 2018.

[26] C. Davini, A. Morassi, and N. Rovere, "Modal analysis of notched bars: tests and comments on the sensitivity of an identification technique," Journal of Sound and Vibration, vol. 179, no. 3, pp. 513-527, 1995.

[27] S. Khatir, K. Dekemele, M. Loccufier, T. Khatir, and M. Abdel Wahab, "Crack identification method in beam-like structures using changes in experimentally measured frequencies and Particle Swarm Optimization," Comptes Rendus Mécanique, vol. 346, no. 2, pp. 110-120, 2018.

[28] M. Abravesh, A. Sheikholeslami, H. Abravesh, and M. Y. Asrami, "Estimation of parameters of metal-oxide surge arrester models using big bang-big crunch and hybrid big bang-big crunch algorithms," Journal of Artificial Intelligence and Data Mining, vol. 4, no. 2, 2016.

[29] Z. H. Zhan and J. Zhang, "Adaptive particle swarm optimization," Ant Colony Optimization and Swarm Intelligence, vol. 50, pp. 227-234, 2009. 